\title{
Diagnóstico e tratamento cirúrgico do teratoma intrapericárdico
}

\author{
Carlos R. MORAES ${ }^{*}$, Sandra MATTOS*, Jorge V. RODRIGUES ${ }^{*}$, Cleuza Lapa SANTOS ${ }^{*}$, Cláudio \\ A. GOMES ${ }^{\star}$, Euclides TENÓRIO*, Fernando MORAES NETO*, Sheila HAZIN*
}

RBCCV 44205-288

MORAES, C. R.; MATTOS, S.; RODRIGUES, J.V.; SANTOS, C. L.; GOMES, C. A.; TENÓRIO, E.; MORAES NETO, F. ; HAZIN, S. - Diagnóstico e tratamento cirúrgico do teratoma intrapericárdico. Rev. Bras. Cir. Cardiovasc., 11 (1): 44-47, 1996.

RESUMO: Descrevemos 2 casos de teratoma intrapericárdico, tumor cardiaco primário raro, usualmente encontrado em neonatos e lactentes e que pode causar insuficiência respiratória, grande acúmulo de líqūido pericárdico e compressão cardiaca, levando à morte no período intra-uterino ou neonatal. Em ambos os casos, o diagnóstico foi estabelecido pelo ecocardiograma realizado em criança de 3 meses com sinais de tamponamento cardiaco e no feto de uma gestante no curso da $38^{\prime \prime}$ semana de gravidez. Ressecção cirúrgica com sucesso em ambos os pacientes foi realizada nas idades de 3 meses e 3 dias de vida, respectivamente. A histologia confirmou o diagnóstico de teratoma. Enfatiza-se a acurácia do diagnóstico ecocardiográfico nestes casos e a importância da indicação cirúrgica precoce.

DESCRITORES: Tumores do coraçāo, cirurgia. Teratoma intrapericárdico, cirurgia.

\section{INTRODUÇÃO}

O teratoma intrapericárdico é tumor cardíaco primário raro que provoca desconforto respiratório, grande acúmulo de líqüido pericárdico e compressão cardiaca, podendo determinar a morte de neonatos e lactentes jovens, se o diagnóstico e a cirurgia precoces não forem realizados $6-8$. O tumor é usualmente benigno, isolado e aderido à aorta ascendente ${ }^{6}$. Diagnóstico diferencial com cisto broncogênico intrapericárdico só pode ser estabelecido através da microscopia ${ }^{3}$. No presente trabaIho, relatamos 2 casos de teratoma intrapericárdico, um dos quais teve o diagnóstico e o planejamento cirúrgico estabelecidos ainda na vida intra-uterina.

\section{RELATO DOS CASOS}

\section{Caso de $n^{\circ} 1$}

Criança do sexo feminino, com 3 meses de vida, pesando $4800 \mathrm{gr}$ foi encaminhada ao Instituto do Coração de Pernambuco (Ficha 2685) por apresentar dispnéia progressiva desde os dois meses. Nascida a termo, de parto eutócico. Ao exame físico, apresentava-se em franca insuficiência cardiaca congestiva. Exibia dispnéia +++ , os pulsos eram palpáveis e simétricos, o precórdio abaulado e não se palpavam frêmitos. O ritmo cardíaco era regular com 140 bpm. Bulhas hipofonéticas, sem sopros. Pulmões limpos. Fígado palpável $6 \mathrm{~cm}$ abaixo da

\footnotetext{
Trabalho realizado no Instituto do Coraçāo de Pernambuco. Real Hospital Português de Beneficência em Pernambuco. Recife, PE, Brasil. Recebido para publicação em janeiro de 1995.

* Do Instituto do Coração de Pernambuco.

Endereço para correspondência: Carlos R. Moraes. Av. Portugal, 163. CEP: 52010-010 Recife, PE, Brasil.
} 
MORAES, C. R.; MATTOS, S.; RODRIGUES, J. V.; SANTOS, C. L.; GOMES, C. A.; TENÓRIO, E.; MORAES NETO, F. ; HAZIN, S. - Diagnóstico e tratamento cirúrgico do teratoma intrapericárdico. Rev. Bras. Cir. Cardiovasc., 11 (1):44-47, 1996.

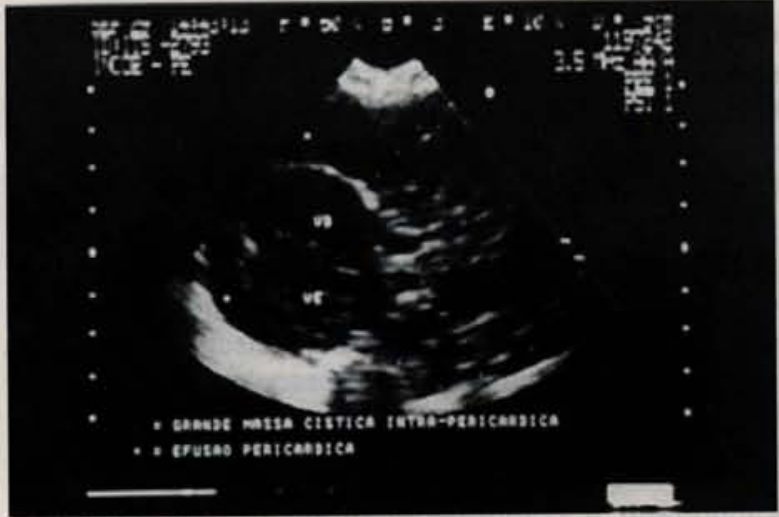

Fig. 1- Ecocardiograma do caso 1 observando-se grande quantidade líquiido pericárdico e o tumor localizado na região do pedículo vascular.

borda costal direita. A radiografia do tórax exibia área cardíaca ++++. Ecocardiograma biplano revelou anatomia intracardíaca normal, volumoso derrame pericárdico e presença de massa tumoral, ocupando a região do pedículo vascular. Estabelecido o diagnóstico de tumor intrapericárdico, indicou-se a cirurgia. Em 5.10.93, a criança foi submetida a esternotomia mediana. Aberto o pericárdio, aspirouse grande quantidade de líqüido citrino. Havia um tumor medindo $7,5 \times 5,5 \mathrm{~cm}$, fortemente aderido aos átrios direito e esquerdo e, especialmente, à aorta ascendente. $O$ tumor foi inteiramente ressecado. O coração era de tamanho e aspecto normais e ficou livre dentro de uma grande cavidade pericárdica. $O$ estudo histológico do tumor revelou tratar-se de teratoma cístico benigno. A paciente teve uma excelente evolução pós-operatória. Decorridos dois anos, a criança encontra-se assintomática. Radiografia do tórax e ecocardiograma são normais.

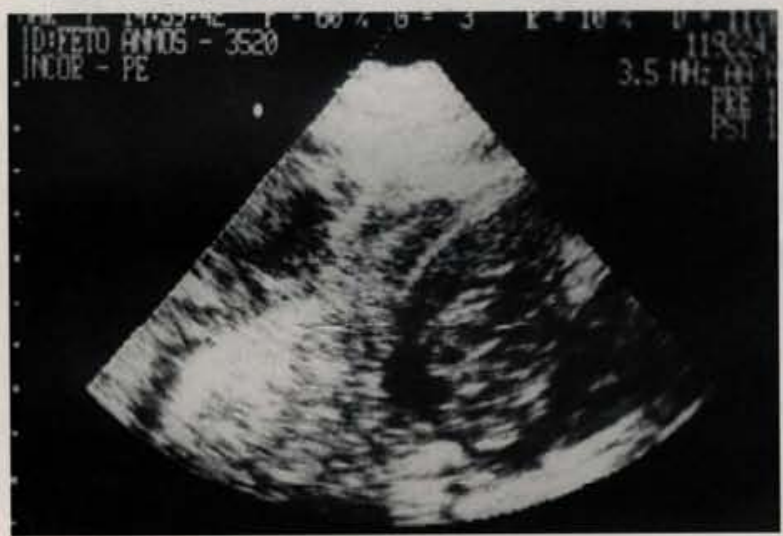

Fig. 2 - Ecocardiograma fetal do caso 2. Nota-se o coração do feto e o tumor intra-pericárdico.

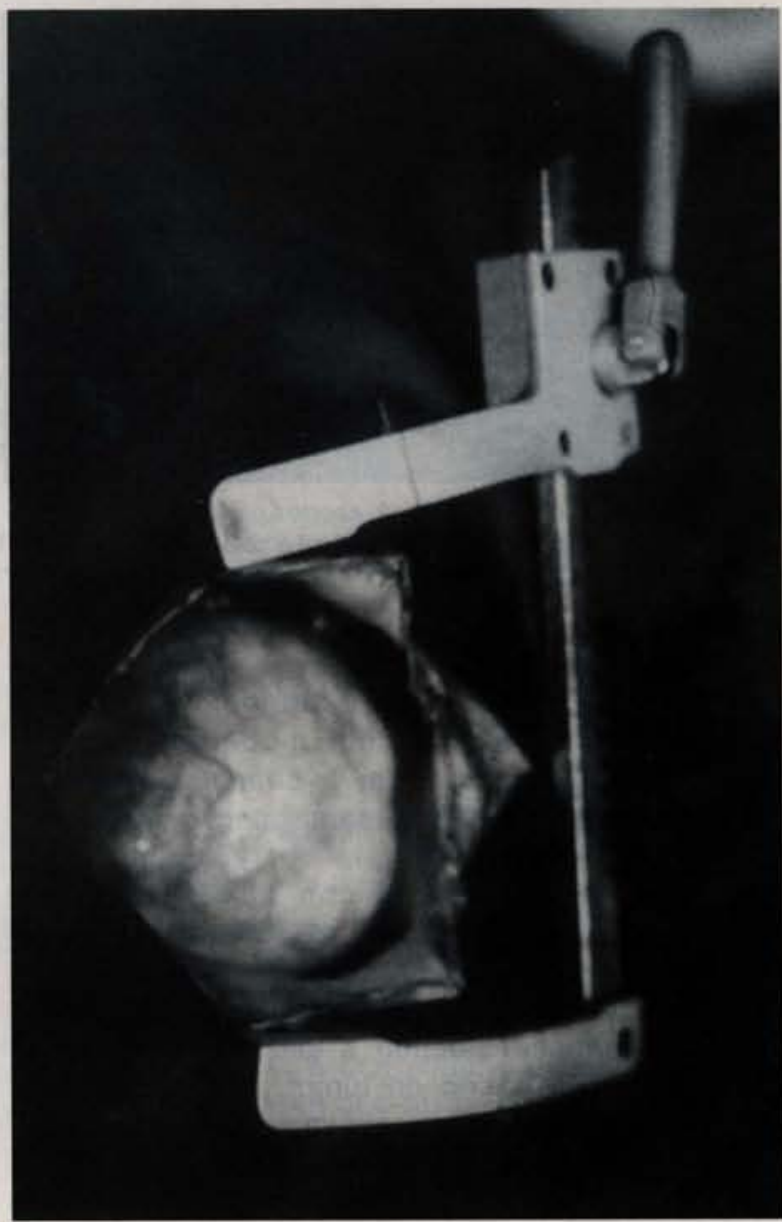

Fig. 3 - O tumor ressecado do caso 2.

\section{Caso de $n^{\circ} 2$}

Gestante de 21 anos foi submetida a ecocardiografia fetal no Instituto do Coração de Pernambuco haja vista a ultra-sonografia de rotina, realizada na $36^{\text {a }}$ semana da gestação ter revelado "massa de ecogenicidade heterogênea no mediastino". Observou-se nesse exame anatomia intracardiaca normal, derrame pericárdico moderado e grande massa cística intrapericárdica anterior e bem próxima aos grandes vasos. O diagnóstico de tumor intrapericárdico (provável teratoma) foi estabelecido, e a gestante foi esclarecida sobre o significado dos achados e a necessidade de acompanhamento do feto e provável manuseio cirúrgico neonatal. $\mathrm{Na}$ $40^{\mathrm{a}}$ semana de gestação, já atingida a maturidade pulmonar fetal, realizou-se parto cesareano. $O$ recém-nascido, do sexo masculino, veio a termo em boas condições, pesando $3400 \mathrm{gr}$. Avaliação clínica neonatal foi normal. Eletrocardiograma sem alterações, normal; a radiografia do tórax mostraya gran- 
MORAES, C. R.; MATTOS, S.; RODRIGUES, J. V.; SANTOS, C. L.; GOMES, C. A.; TENÓRIO, E.; MORAES NETO, F. ; HAZIN, S. - Diagnóstico e tratamento cirúrgico do teratoma intrapericárdico. Rev. Bras. Cir. Cardiovasc., 11 (1):44-47, 1996.

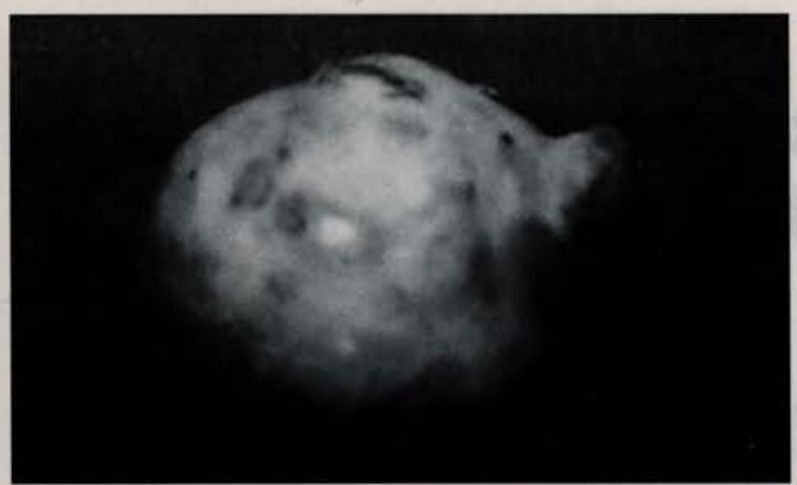

Fig. 4- Aspecto cirúrgico do caso 2. Aberto o pericárdio, visibiliza-se o tumor que esconde e comprime o coração.

de aumento da área cardíaca e a ecocardiografia confirmou os achados intra-uterinos de imagem cística intrapericárdica com moderada coleção líqüida. No $3^{\circ}$ dia de vida, a criança apresentou desconforto respiratório e regurgitação tricúspide. Neste mesmo dia (24.3.94), foi operada por meio de esternotomia mediana (Ficha 2843). Aberto o pericárdio, aspirou-se cerca de $30 \mathrm{ml}$ de líqüido serossanguinolento. Havia um tumor medindo $4,5 \mathrm{x}$ $3,5 \mathrm{~cm}$, localizado entre a veia cava superior e a aorta e fortemente aderido a este vaso. Realizouse a completa excisão do tumor. A evolução pósoperatória decorreu sem qualquer complicação. O estudo histológico revelou tratar-se de teratoma cístico benigno. Um ano e meio após operação, a criança está assintomática, com radiografia do tórax e ecocardiograma normais.

Teratomas são neoplasmas que contêm tecido endodérmico, mesodérmico e neuroectodérmico. Podem ser encontrados no cérebro, pescoço, tórax, retroperitônio, testículos, ovários e região sacrococcígea. Teratoma intrapericárdico é raro, de incidência incerta, não existindo preponderância de sexo. Ocorre mais freqüentemente em recém-nascidos e lactentes jovens. O tumor é geralmente bem encapsulado, de coloração acinzentada, consistência variável e multicístico. Derrame pericárdico, normalmente maciço, é encontrado na maioria dos pacientes. Essas mesmas características são exibidas pelo cisto broncogênico intrapericárdico, e o diagnóstico diferencial só pode ser feito pela histologia, visto que esses tumores derivam do aparelho respiratório e mucosa digestiva, enquanto os teratomas se originam dos três folhetos embrionários. O teratoma intrapericárdico é usualmente benigno e único, estando fortemente aderido à aorta ascendente, de onde recebe sua vascularização.

Os sintomas surgem precocemente. Desconforto respiratório é o sintoma mais importante, sendo secundário à compressão brônquica ou pulmonar. Teratoma intrapericárdico deve ser suspeitado em qualquer criança que apresente desconforto respiratório, cianose, obstrução de veia cava superior ou evidência de tamponamento cardíaco. A tumoração e o grande derrame pericárdico, sempre presente, são evidenciados radiologicamente por aumento da área cardíaca. Trabalhos prévios têm utilizado a radiografia do tórax em combinação com ecocardiografia, tomografia computadorizada e angiocardiografia no diagnóstico desses tumores. Em nossos 2 pacientes, a ecocardiografia bidimensional mostrou ser método extremamente acurado no diagnóstico do teratoma intrapericárdico, e nenhum outro procedimento foi necessário. Ressalte-se que em 1 deles o diagnóstico intra-uterino foi estabelecido sem dificuldades através da ecocardiografia fetal, possibilitando a gestação monitorizada e os períodos pré, peri e pós-natal programados. Assim, a criança foi operada no $3^{\circ}$ dia, quando o quadro clínico começou a deteriorar.

Evidentemente, o tratamento cirúrgico se impõe tão logo a diagnóstico de teratoma ou de tumor intrapericárdico seja estabelecido. A cirurgia é feita sem auxílio de circulação extracorpórea, visto que as aderências do tumor com o coração, especialmente com a aorta ascendente, podem ser desfeitas sem dificuldades. Tal como foi observado, ocorre regressão imediata dos sintomas após a operação e o prognóstico é excelente, dada a natureza usualmente benigna do tumor. 
MORAES, C. R.; MATTOS, S.; RODRIGUES, J. V.; SANTOS, C. L.; GOMES, C. A.; TENÓRIO, E.; MORAES NETO, F. ; HAZIN, S. - Diagnóstico e tratamento cirúrgico do teratoma intrapericárdico. Rev. Bras. Cir. Cardiovasc., 11 (1):44-47, 1996.

RBCCV 44205-288

MORAES, C. R.; MATTOS, S.; RODRIGUES, J.V.; SANTOS, C. L.; GOMES, C. A.; TENÓRIO, E.; MORAES NETO, F. ; HAZIN, S. - Diagnosis and surgical treatment of intrapericardial teratoma. Rev. Bras. Cir. Cardiovasc., 11 (1): 44-47, 1996.

ABSTRACT: We report two cases of intrapericardial teratoma, a rare cardiac tumor, usually found in neonates and young infants, which may cause respiratory distress, large pericardial effusions and cardiac compression leading to intra-uterine or neonatal death. Both cases were diagnosed noninvasively by echocardiogram, in a 3 month-old girl with signs of cardiac tamponade, and by fetal echocardiography, in a 38 week pregnant woman. Successful surgical removal of the tumor was undertaken in both patients at the ages of 3 months and 3 days of life, respectively. Histology confirmed the diagnosis of teratoma. It is emphasized the diagnostic accuracy of achocardiography and the importance of early surgical treatment in the management of intrapericardial teratoma.

DESCRIPTORS: Heart tumors, surgery. Intrapericardial teratoma, surgery.

\section{REFERÊNCIAS BIBLIOGRÁFICAS}

1 ALDOUSANY, A. W.; JOYNER, J. C.; PRICE, R. A.; BOULDEN, T.; WATSON, D.; DISESSA, T. G. Diagnosis and treatment of intrapericardial teratoma. J. Pediatr., 8: 51-53, 1987.

2 ARCINIEGAS, E.; HAKIMI, M.; FAROOKI, Z. Q.; GREEN, E. W. - Intrapericardial teratoma in infancy. J. Thorac. Cardiovasc. Surg., 79: 306-311, 1980.

3 DEENADAYALU, R. P.; TUURI, D.; DE WALL, R. A.; JOHNSON, G. F. - Intrapericardial teratoma and broncogenic cyst. J. Thorac. Cardiovasc. Surg., 67: 945-951, 1974.

4 DEGEETER, B.; KRETZ, J. G.; NISAND, I.; EISENMANN, B.; KIENY, M. T.; KIENY, R. Intrapericardial teratoma in a newborn infant: use of fetal echocardiography. Ann. Thorac. Surg., 35: 664666, 1983.

5 GROVES, A. M. M.; FAGG, N. L. K.; COOK, A. C.; ALLAN, L. D. - Cardiac tumors in intrauterine life. Arch. Dis. Childhood, 67: 1189-1192, 1992.

6 LEGNAMI, F. A. \& CORWIN, R. D. - Intrapericardial teratoma: report of a case. Am. Heart J. 65: 674676, 1963.

7 LUBIN, B. H.; FRIEDMAN, S.; MILLER, N. Intrapericardial teratoma associated with pericardial effusion: an surgical acute problem in infancy. $\mathrm{J}$. Pediatr. Surg., 2: 336-342, 1967.

8 ZERELLA, J. T. \& HALPE, D. C. - Intrapericardial teratoma: neonatal cardiorespiratory distress amenable by surgery. J. Pediatr. Surg., 15: 961$963,1980$. 